\title{
Stabilizing the long-time behavior of the Navier-Stokes-Voigt equations by fast oscillating-in-time forces
}

by

\author{
Cung The ANH and Vu Manh TOI \\ Presented by Piotr BILER
}

Summary. We show that under the action of fast oscillating-in-time external forces, there exists a unique time periodic solution, and any weak solution to the Navier-Stokes-Voigt equations converges to this time periodic flow with exponential speed in time.

1. Introduction. Let $\mathcal{O}$ be a (not necessarily bounded) domain satisfying the Poincaré inequality in $\mathbb{R}^{3}$ with smooth boundary $\partial \mathcal{O}$. We consider the following Navier-Stokes-Voigt equations:

$$
\begin{cases}y_{t}-\alpha^{2} \Delta y_{t}-\nu \Delta y+(y \cdot \nabla) y+\nabla p=F, & x \in \mathcal{O}, t>0, \\ \nabla \cdot y=0, & x \in \mathcal{O}, t>0, \\ y=0, & x \in \partial \mathcal{O}, t>0,\end{cases}
$$

where $y=y(x, t)$ is the unknown velocity and $p=p(x, t)$ is the unknown pressure, $\nu>0$ is the kinematic viscosity coefficient, $\alpha$ is a length scale parameter characterizing the elasticity of the fluid, and $F$ is the external force.

The system (1.1) was introduced by Oskolkov [13] as a model of motion of certain linear viscoelastic fluids. The system (1.1) was also proposed by Cao, Lunasin and Titi [5] as a regularization, for small values of $\alpha$, of the

2010 Mathematics Subject Classification: 76A10, 93D15, 35Q35, 93C20.

Key words and phrases: Navier-Stokes-Voigt equations, fast oscillating force, time periodic solution, stabilization.

Received 28 December 2016; revised 24 July 2017.

Published online 23 October 2017. 
3D Navier-Stokes equations for the sake of direct numerical simulations. The presence of the regularizing term $-\alpha^{2} \Delta u_{t}$ leads to the fact that system (1.1) has a natural energy inequality in $H^{1}$-norm, and this has many important consequences. On the one hand, it leads to the global well-posedness of (1.1) both forward and backward in time, even in three dimensions. On the other hand, it changes the parabolic character of the limit Navier-Stokes equations, so the Navier-Stokes-Voigt system behaves like a damped hyperbolic system. In fact, the Navier-Stokes-Voigt system belongs to the class of so-called $\alpha$-models in fluid mechanics (see e.g. [10]), but it has an attractive advantage over other $\alpha$-models in that one does not need to impose any additional artificial boundary condition (besides the Dirichlet boundary conditions) to get global well-posedness. We also refer the reader to [8] for an interesting application of the Navier-Stokes-Voigt equations in image inpainting.

In the last few years, mathematical questions related to 3D NavierStokes-Voigt equations have attracted the attention of a number of mathematicians. The existence and long-time behavior of solutions in terms of existence of attractors to the Navier-Stokes-Voigt equations in domains that are bounded or unbounded but satisfying the Poincaré inequality were investigated extensively in [1, 4, 9, 11, 14. The decay rate and decay characterization of solutions to the Navier-Stokes-Voigt equations on the whole space were studied more recently in [2, 12, 15].

As in the case of Navier-Stokes equations, one can easily show that if the external force is time-independent and small enough in norm, then there exists a unique stationary solution to the Navier-Stokes-Voigt equations, and this solution is globally exponentially stable.

The aim of this paper is to study the effect of fast oscillating-in-time forces on the long-time behavior of solutions to Navier-Stokes-Voigt equations. Using some ideas of [7], we show that if the external force is oscillating fast enough in time but does not necessarily have small spatial norms, then there exists a unique time periodic solution such that any solution to the Navier-Stokes-Voigt equations converges to that solution with exponential speed in time. For results on feedback stabilization of a given time periodic solution to Navier-Stokes equations, we refer the reader to the recent monograph of Barbu [3]. Notice that all our results are also valid in the case of periodic boundary conditions as in [7]; however, here we consider the Dirichlet boundary conditions because they have more physical meaning. We also emphasize that we do not need to require the boundedness of the domain $\mathcal{O}$ considered.

Let us denote

$$
\mathcal{V}=\left\{y \in C_{0}^{\infty}(\mathcal{O})^{3}: \nabla \cdot y=0\right\}
$$


In order to study the system (1.1), we use the function space

$$
H=\overline{\mathcal{V}}^{L^{2}(\mathcal{O})^{3}}:=\text { the closure of } \mathcal{V} \text { in } L^{2}(\mathcal{O})^{3}
$$

with the norm

$$
|y|=\left(\sum_{j=1}^{3} \int_{\mathcal{O}}\left|y_{j}\right|^{2} d x\right)^{1 / 2}
$$

and

$$
V=\overline{\mathcal{V}}^{H_{0}^{1}(\mathcal{O})^{3}}:=\text { the closure of } \mathcal{V} \text { in } H_{0}^{1}(\mathcal{O})^{3}
$$

with the norm

$$
\|y\|=\left(\int_{\mathcal{O}}|\nabla y|^{2} d x\right)^{1 / 2}
$$

Let $P$ be the Helmholtz-Leray orthogonal projection in $L^{2}(\mathcal{O})^{3}$ onto the space $H$. We also define the bilinear form

$$
B(u, v):=P((u \cdot \nabla) v)
$$

and the trilinear form

$$
b(u, v, w)=\int_{\mathcal{O}} B(u, v) \cdot v d x,
$$

for all $u, v, w \in V$. We see that

$$
b(u, v, v)=0 \quad \text { for all } u, v \in V .
$$

We have the following estimate (see, e.g., [6]):

$$
|b(u, v, w)| \leq c_{0}\|u\|\|v\||w|^{1 / 2}\|w\|^{1 / 2}, \quad \forall u, v, w \in V .
$$

Because of the assumption on the domain $\mathcal{O}$, we have the Poincaré inequality

$$
\|v\|^{2} \geq \lambda_{1}|v|^{2} \quad \text { for all } v \in V
$$

for some $\lambda_{1}>0$. It is known that if $\mathcal{O}$ is bounded, we can take for $\lambda_{1}$ the first eigenvalue of the Stokes operator $A=-P \Delta$, which is defined by

$$
\langle A u, v\rangle=\sum_{i=1}^{3} \int_{\mathcal{O}} \nabla u_{i} \cdot \nabla v_{i} d x, \quad u, v \in V .
$$

Here $\langle\cdot, \cdot\rangle$ denotes the duality between $V$ and its dual space $V^{\prime}$.

We now make the following assumption on the external force.

Assumption (F). For any positive constant $\Omega>0$, the force term $F(x, \Omega t)$ is a time periodic function with period $T_{\mathrm{per}}$, having the following structure: There exists a time periodic function $G(x, \Omega t)$ with period $T_{\mathrm{per}}$ 
such that

$$
\begin{cases}\frac{1}{\Omega}\left(\partial_{t} G(x, \Omega t)-\alpha^{2} \Delta \partial_{t} G(x, \Omega t)\right)=-F(x, \Omega t), & x \in \mathcal{O}, t>0 \\ \nabla \cdot G=0, & x \in \mathcal{O}, t>0 \\ G=0, & x \in \partial \mathcal{O}, t>0\end{cases}
$$

Further,

$$
F \in L^{\infty}\left(0, T_{\mathrm{per}} ; H\right) \text {, and }\|F\|_{L^{\infty}\left(0, T_{\mathrm{per}} ; H\right)} \text { is independent of } \Omega .
$$

Moreover, $G \in L^{\infty}\left(0, T_{\mathrm{per}} ; V\right)$ and there exists a positive constant $\mathcal{G}$ independent of $\Omega$ such that

$$
\|G\|_{L^{\infty}\left(0, T_{\mathrm{per}} ; V\right)}^{2} \leq \mathcal{G}\|F\|_{L^{\infty}\left(0, T_{\mathrm{per}} ; H\right)}^{2} .
$$

Let us give an example of an external force $F$. Let

$F(x, \Omega t)=f(x) \sin \Omega t, \quad$ where $f \in H$ and $\Omega$ is a positive constant.

We will find $G(x, \Omega t)$ of the form

$$
G(x, \Omega t)=-h(x) \cos \Omega t .
$$

Substituting $G$ into 1.4 , we get

$$
\begin{cases}\left(I-\alpha^{2} \Delta\right) h(x)=f(x), & x \in \mathcal{O}, \\ \nabla \cdot h=0, & x \in \mathcal{O}, \\ h=0, & x \in \partial \mathcal{O} .\end{cases}
$$

Then, by the Lax-Milgram lemma on the Hilbert space $V$, we get the existence and uniqueness of $h \in V$ such that

$$
\|h\|^{2} \leq \mathcal{G}|f|^{2}
$$

for some positive constant $\mathcal{G}$ independent of $\Omega$. Thus $G \in L^{\infty}\left(0, T_{\text {per }} ; V\right)$ and $G$ satisfies (1.5), where $T_{\text {per }}=2 \pi / \Omega$.

More generally, we can take $F(x, \Omega t)=f(x) \varphi(\Omega t)$, where $f \in H$ and $\varphi(t)$ is a real-valued periodic continuous function with period $T$. Then $F$ satisfies Assumption (F) with $T_{\mathrm{per}}=T / \Omega$.

2. Main results. First, we prove the existence of a time periodic solution to system (1.1).

Theorem 2.1. Let $F$ satisfy Assumption (F). Then there exists $\Omega_{0}>0$ depending on $\nu, c_{0}, \alpha, \lambda_{1}, \mathcal{G}$ and $\|F\|_{L^{\infty}\left(0, T_{\mathrm{per}} ; H\right)}$ such that for any $\Omega \geq \Omega_{0}$, system (1.1) has a $T_{\mathrm{per}}$-periodic solution $y_{\mathrm{per}}$ satisfying

$$
\left|y_{\text {per }}(t)\right|^{2}+\alpha^{2}\left\|y_{\text {per }}(t)\right\|^{2} \leq \frac{\nu^{2} \alpha}{2 c_{0}^{2}}, \quad t \in\left[0, T_{\text {per }}\right] .
$$


Proof. Since under Assumption (F) we have $F \in L^{\infty}\left(0, T_{\text {per }} ; H\right) \subset$ $L^{2}\left(0, T_{\text {per }} ; V^{\prime}\right)$, by [1, Theorem 3.2] for each initial datum $y^{0} \in V$ there exists a unique weak solution $y \in C\left(\left[0, T_{\mathrm{per}}\right] ; V\right)$ to (1.1) satisfying the initial condition $y(0)=y^{0}$.

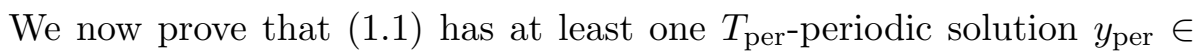
$L^{\infty}\left(0, T_{\text {per }} ; V\right)$ satisfying (2.1). To do this, set

$$
y=Y-\frac{1}{\Omega} G(x, \Omega t) .
$$

Then we deduce from 1.1 that

$$
\begin{cases}Y_{t}-\alpha^{2} \Delta Y_{t}-\nu \Delta Y+(Y \cdot \nabla) Y+\nabla p & \\ =\frac{1}{\Omega}\left(\partial_{t} G(x, \Omega t)-\alpha^{2} \Delta \partial_{t} G(x, \Omega t)\right)+F(x, \Omega t) & \\ \quad-\frac{\nu}{\Omega} \Delta G-\frac{1}{\Omega^{2}}(G \cdot \nabla) G+\frac{1}{\Omega}[(Y \cdot \nabla) G+(G \cdot \nabla) Y], & x \in \mathcal{O}, t>0 \\ \nabla \cdot Y=0, & x \in \mathcal{O}, t>0, \\ Y=0, & x \in \partial \mathcal{O}, t>0 .\end{cases}
$$

If we use (1.4) then 2.3 becomes

$$
\begin{cases}Y_{t}-\alpha^{2} \Delta Y_{t}-\nu \Delta Y+(Y \cdot \nabla) Y+\nabla p & \\ \quad=-\frac{\nu}{\Omega} \Delta G-\frac{1}{\Omega^{2}}(G \cdot \nabla) G+\frac{1}{\Omega}[(Y \cdot \nabla) G+(G \cdot \nabla) Y], & x \in \mathcal{O}, t>0, \\ \nabla \cdot Y=0, & x \in \mathcal{O}, t>0, \\ Y=0, & x \in \partial \mathcal{O}, t>0 .\end{cases}
$$

Multiplying (2.4) by $Y$ and integrating over $\mathcal{O}$ we get

$$
\begin{aligned}
\frac{1}{2} \frac{d}{d t}\left(|Y(t)|^{2}\right. & \left.+\alpha^{2}\|Y(t)\|^{2}\right)+\nu\|Y(t)\|^{2} \\
& =-\frac{\nu}{\Omega} \int_{\mathcal{O}} \Delta G \cdot Y d x-\frac{1}{\Omega^{2}} b(G, G, Y)+\frac{1}{\Omega} b(Y, G, Y) .
\end{aligned}
$$

Here we have used the fact that $b(G, Y, Y)=0$.

Integrating by parts and using the Cauchy inequality, we obtain

$$
\begin{aligned}
-\frac{\nu}{\Omega} \int_{\mathcal{O}} \Delta G \cdot Y d x & =\frac{\nu}{\Omega} \int_{\mathcal{O}} \nabla G \cdot \nabla Y d x \\
& \leq \frac{\nu}{8}\|Y(t)\|^{2}+\frac{2 \nu}{\Omega^{2}}\|G\|_{L^{\infty}\left(0, T_{\mathrm{per}} ; V\right)}^{2} .
\end{aligned}
$$


Using (1.2), 1.3) and (1.5), we get

$$
\begin{aligned}
\frac{1}{\Omega} b(Y, G, Y) & \leq \frac{c_{0}}{\lambda_{1}^{1 / 4} \Omega}\|G\|_{L^{\infty}\left(0, T_{\mathrm{per}} ; V\right)}\|Y(t)\|^{2} \\
& \leq \frac{c_{0} \sqrt{\mathcal{G}}}{\lambda_{1}^{1 / 4} \Omega}\|F\|_{L^{\infty}\left(0, T_{\mathrm{per}} ; H\right)}\|Y(t)\|^{2} .
\end{aligned}
$$

Using 1.2, 1.3 and the Cauchy inequality, we obtain

$$
\begin{aligned}
-\frac{1}{\Omega^{2}} b(G, G, Y) & \leq \frac{c_{0}}{\lambda_{1}^{1 / 4} \Omega^{2}}\|G\|_{L^{\infty}\left(0, T_{\mathrm{per}} ; V\right)}^{2}\|Y(t)\| \\
& \leq \frac{\nu}{8}\|Y(t)\|^{2}+\frac{2 c_{0}^{2}}{\nu \lambda_{1}^{1 / 2} \Omega^{4}}\|G\|_{L^{\infty}\left(0, T_{\mathrm{per}} ; V\right)}^{4} .
\end{aligned}
$$

Taking

$$
\Omega \geq \frac{4 c_{0} \sqrt{\mathcal{G}}}{\nu \lambda_{1}^{1 / 4}}\|F\|_{L^{\infty}\left(0, T_{\mathrm{per}} ; H\right)},
$$

from (2.5)-2.8 we obtain

$$
\begin{aligned}
\frac{d}{d t}\left(|Y(t)|^{2}+\alpha^{2} \| Y(t)\right. & \left.\|^{2}\right)+\nu\|Y(t)\|^{2} \\
& \leq \frac{4 \nu}{\Omega^{2}}\|G\|_{L^{\infty}\left(0, T_{\mathrm{per}} ; V\right)}^{2}+\frac{4 c_{0}^{2}}{\nu \lambda_{1}^{1 / 2} \Omega^{4}}\|G\|_{L^{\infty}\left(0, T_{\mathrm{per}} ; V\right)}^{4} .
\end{aligned}
$$

Hence, using the Poincaré inequality 1.3 and 1.5 , we get

$$
\begin{aligned}
\frac{d}{d t}\left(|Y(t)|^{2}+\right. & \left.\alpha^{2}\|Y(t)\|^{2}\right)+d_{0}\left(|Y(t)|^{2}+\alpha^{2}\|Y(t)\|^{2}\right) \\
\leq & \frac{\mathcal{G}\|F\|_{L^{\infty}\left(0, T_{\mathrm{per}} ; H\right)}^{2}}{\Omega^{2}}\left(4 \nu+\frac{4 c_{0}^{2} \mathcal{G}\|F\|_{L^{\infty}\left(0, T_{\mathrm{per}} ; H\right)}^{2}}{\nu \lambda_{1}^{1 / 2} \Omega^{2}}\right),
\end{aligned}
$$

where

$$
d_{0}=\frac{\nu}{2} \min \left\{\frac{1}{\alpha^{2}}, \lambda_{1}\right\} .
$$

Multiplying $(2.9)$ by $e^{d_{0} t}$ and then integrating from 0 to $t$ yields

$$
\begin{aligned}
|Y(t)|^{2} & +\alpha^{2}\|Y(t)\|^{2} \leq e^{-d_{0} t}\left(|Y(0)|^{2}+\alpha^{2}\|Y(0)\|^{2}\right) \\
& +\left(1-e^{-d_{0} t}\right) \frac{\mathcal{G}\|F\|_{L^{\infty}\left(0, T_{\mathrm{per}} ; H\right)}^{2}}{d_{0} \Omega^{2}}\left(4 \nu+\frac{4 c_{0}^{2} \mathcal{G}\|F\|_{L^{\infty}\left(0, T_{\mathrm{per}} ; H\right)}^{2}}{\nu \lambda_{1}^{1 / 2} \Omega^{2}}\right) .
\end{aligned}
$$

Set

$$
R^{2}=\frac{\nu^{2} \alpha}{6 c_{0}^{2}}
$$


We will prove that for any $Y(0)$ such that $|Y(0)|^{2}+\alpha^{2}\|Y(0)\|^{2} \leq R^{2}$ we also have $\left|Y\left(T_{\text {per }}\right)\right|^{2}+\alpha^{2}\left\|Y\left(T_{\text {per }}\right)\right\|^{2} \leq R^{2}$. Indeed, from (2.10) we have

$$
\begin{aligned}
& \left|Y\left(T_{\mathrm{per}}\right)\right|^{2}+\alpha^{2}\left\|Y\left(T_{\mathrm{per}}\right)\right\|^{2} \leq e^{-d_{0} T_{\mathrm{per}}} R^{2} \\
& \quad+\left(1-e^{-d_{0} T_{\mathrm{per}}}\right) \frac{\mathcal{G}\|F\|_{L^{\infty}\left(0, T_{\mathrm{per}} ; H\right)}^{2}}{d_{0} \Omega^{2}}\left(4 \nu+\frac{4 c_{0}^{2} \mathcal{G}\|F\|_{L^{\infty}\left(0, T_{\mathrm{per}} ; H\right)}^{2}}{\nu \lambda_{1}^{1 / 2} \Omega^{2}}\right) .
\end{aligned}
$$

Then from 2.11) we get, when $\Omega \geq \Omega_{0}>0$ is large enough,

$$
\left|Y\left(T_{\mathrm{per}}\right)\right|^{2}+\alpha^{2}\left\|Y\left(T_{\mathrm{per}}\right)\right\|^{2} \leq R^{2} .
$$

Since the ball $\left\{|\cdot|^{2}+\alpha^{2}\|\cdot\|^{2} \leq R^{2}\right\} \subset V$ is a convex set in a Hilbert space, it is compact in the weak topology. Hence, by using the Tychonoff theorem we conclude that the map $Y(0) \mapsto Y\left(T_{\text {per }}\right)$ has a fixed point $Y^{*}$. Then the solution $Y_{\text {per }}$ of system (2.4) with initial datum $Y^{*}$ is a $T_{\text {per-periodic solution }}$ satisfying

$$
\left|Y_{\text {per }}(0)\right|^{2}+\alpha^{2}\left\|Y_{\text {per }}(0)\right\|^{2}=\left|Y_{\text {per }}\left(T_{\text {per }}\right)\right|^{2}+\alpha^{2}\left\|Y_{\text {per }}\left(T_{\text {per }}\right)\right\|^{2} \leq R^{2} .
$$

Moreover, from (2.10), the solution $Y_{\text {per }}$ satisfies

$$
\begin{aligned}
& \left|Y_{\mathrm{per}}(t)\right|^{2}+\alpha^{2}\left\|Y_{\mathrm{per}}(t)\right\|^{2} \leq e^{-d_{0} t} R^{2} \\
& \quad+\left(1-e^{-d_{0} t}\right) \frac{\mathcal{G}\|F\|_{L^{\infty}\left(0, T_{\mathrm{per}} ; H\right)}^{2}}{d_{0} \Omega^{2}}\left(4 \nu+\frac{4 c_{0}^{2} \mathcal{G}\|F\|_{L^{\infty}\left(0, T_{\mathrm{per}} ; H\right)}^{2}}{\nu \lambda_{1}^{1 / 2} \Omega^{2}}\right) .
\end{aligned}
$$

So, when $\Omega \geq \Omega_{0}$ large enough, we infer from 2.12 that

$$
\left|Y_{\mathrm{per}}(t)\right|^{2}+\alpha^{2}\left\|Y_{\mathrm{per}}(t)\right\|^{2} \leq R^{2} \quad \text { for all } t \in\left[0, T_{\mathrm{per}}\right] .
$$

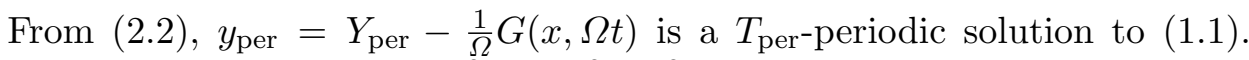
Using the inequality $(a+b)^{2} \leq 2\left(a^{2}+b^{2}\right)$, we obtain

$$
\begin{aligned}
\left|y_{\text {per }}(t)\right|^{2}+\alpha^{2}\left\|y_{\text {per }}(t)\right\|^{2} \leq & 2\left(\left|Y_{\text {per }}(t)\right|^{2}+\alpha^{2}\left\|Y_{\text {per }}(t)\right\|^{2}\right) \\
& +\frac{2}{\Omega^{2}}\left(\|G\|_{L^{\infty}\left(0, T_{\mathrm{per}} ; H\right)}^{2}+\alpha^{2}\|G\|_{L^{\infty}\left(0, T_{\mathrm{per}} ; V\right)}^{2}\right) .
\end{aligned}
$$

Hence using 1.5 we get

$$
\begin{aligned}
\left|y_{\mathrm{per}}(t)\right|^{2}+\alpha^{2}\left\|y_{\mathrm{per}}(t)\right\|^{2} \leq & 2\left(\left|Y_{\mathrm{per}}(t)\right|^{2}+\alpha^{2}\left\|Y_{\mathrm{per}}(t)\right\|^{2}\right) \\
& +\frac{2 \mathcal{G}}{\Omega^{2}}\left(\frac{1}{\lambda_{1}}+\alpha^{2}\right)\|F\|_{L^{\infty}\left(0, T_{\mathrm{per}} ; H\right)}^{2} .
\end{aligned}
$$

Combining (2.13) and (2.14) we deduce that

$$
\left|y_{\text {per }}(t)\right|^{2}+\alpha^{2}\left\|y_{\text {per }}(t)\right\|^{2} \leq 3 R^{2}=\frac{\nu^{2} \alpha}{2 c_{0}^{2}} \quad \text { for all } t \in\left[0, T_{\text {per }}\right],
$$

for $\Omega \geq \Omega_{0}$ large enough. This completes the proof. 
We now prove the global exponential stability of the time periodic solution $y_{\text {per }}$.

TheOREM 2.2. Let $y^{0} \in V$ and suppose the external force $F$ satisfies Assumption (F). Then any weak solution y to system (1.1) with initial datum $y^{0}$ satisfies

$$
\begin{aligned}
\left|y(t)-y_{\mathrm{per}}(t)\right|^{2}+\alpha^{2} \| y(t) & -y_{\mathrm{per}}(t) \|^{2} \\
& \leq e^{-d_{0} t}\left(\left|y^{0}-y_{\mathrm{per}}(0)\right|^{2}+\alpha^{2}\left\|y^{0}-y_{\mathrm{per}}(0)\right\|^{2}\right),
\end{aligned}
$$

where

$$
d_{0}=\frac{\nu}{2} \min \left\{\frac{1}{\alpha^{2}}, \lambda_{1}\right\}
$$

and $y_{\text {per }}$ is the time periodic solution obtained in Theorem 2.1. In particular, the periodic solution $y_{\mathrm{per}}$ is unique.

Proof. The function $v=y-y_{\text {per }}$ satisfies the following system:

$$
\begin{cases}v_{t}-\alpha^{2} \Delta v_{t}-\nu \Delta v+\left(y_{\mathrm{per}} \cdot \nabla\right) v+(v \cdot \nabla) y_{\mathrm{per}}+\nabla p=0, & x \in \mathcal{O}, t>0, \\ \nabla \cdot v=0, & x \in \mathcal{O}, t>0, \\ v=0, & x \in \partial \mathcal{O}, t>0, \\ v(0)=y^{0}-y_{\mathrm{per}}(0), & x \in \mathcal{O} .\end{cases}
$$

Multiplying the first equation by $v$ and integrating over $\mathcal{O}$ yields

$$
\frac{1}{2} \frac{d}{d t}\left(|v|^{2}+\alpha^{2}\|v\|^{2}\right)+\nu\|v\|^{2}=b\left(v, y_{\mathrm{per}}, v\right) .
$$

Here, we have used the fact that $b(y, v, v)=0$. Hence, by using 1.2 and the Cauchy inequality, we get

$$
\begin{aligned}
\frac{1}{2} \frac{d}{d t}\left(|v|^{2}+\alpha^{2}\|v\|^{2}\right)+\nu\|v\|^{2} & \leq c_{0}\|v(t)\|^{2}\left|y_{\mathrm{per}}(t)\right|^{1 / 2}\left\|y_{\mathrm{per}}(t)\right\|^{1 / 2} \\
& \leq \frac{c_{0}}{\sqrt{2 \alpha}} \sqrt{\left|y_{\mathrm{per}}(t)\right|^{2}+\alpha^{2}\left\|y_{\mathrm{per}}(t)\right\|^{2}}\|v(t)\|^{2} .
\end{aligned}
$$

From (2.1) we have

$$
\frac{c_{0}}{\sqrt{2 \alpha}} \sqrt{\left|y_{\mathrm{per}}(t)\right|^{2}+\alpha^{2}\left\|y_{\mathrm{per}}(t)\right\|^{2}} \leq \frac{\nu}{2} \quad \text { for all } t \in\left[0, T_{\mathrm{per}}\right] .
$$

Since $\nu\|v\|^{2} \geq d_{0}\left(|v|^{2}+\alpha^{2}\|v\|^{2}\right)$, we deduce from (2.15) and (2.16) that

$$
\frac{d}{d t}\left(|v|^{2}+\alpha^{2}\|v\|^{2}\right)+d_{0}\left(|v|^{2}+\alpha^{2}\|v\|^{2}\right) \leq 0 .
$$

Therefore,

$$
|v(t)|^{2}+\alpha^{2}\|v(t)\|^{2} \leq e^{-d_{0} t}\left(|v(0)|^{2}+\alpha^{2}\|v(0)\|^{2}\right), \quad \forall t \geq 0 .
$$

The proof is complete. 
Acknowledgements. This research is funded by Vietnam National Foundation for Science and Technology Development (NAFOSTED) under grant number 101.02-2015.10.

\section{References}

[1] C. T. Anh and P. T. Trang, Pullback attractors for 3D Navier-Stokes-Voigt equations in some unbounded domains, Proc. Roy. Soc. Edinburgh Sect. A 143 (2013), 223-251.

[2] C. T. Anh and P. T. Trang, Decay rate of solutions to the 3D Navier-Stokes-Voigt equations in $H^{m}$ spaces, Appl. Math. Lett. 61 (2016), 1-7.

[3] V. Barbu, Stabilization of Navier-Stokes Flows, Comm. Control Engrg. Ser., Springer, London, 2011.

[4] A. O. Celebi, V. K. Kalantarov and M. Polat, Global attractors for 2D Navier-StokesVoight equations in an unbounded domain, Appl. Anal. 88 (2009), 381-392.

[5] Y. Cao, E. M. Lunasin and E. S. Titi, Global well-posedness of the three-dimensional viscous and inviscid simplified Bardina turbulence models, Comm. Math. Sci. 4 (2006), 823-848.

[6] P. Constantin and C. Foiaş, Navier-Stokes Equations, Chicago Lectures in Math., Univ. of Chicago Press, Chicago, 1988.

[7] J. Cyranka, P. B. Mucha, E. S. Titi and P. Zgliczyński, Stabilizing the long-time behavior of the Navier-Stokes equations and damped Euler systems by fast oscillating forces, arXiv:1601.04612v1 (2016).

[8] M. A. Ebrahimi, M. Holst and E. Lunasin, The Navier-Stokes-Voight model for image inpainting, IMA J. Appl. Math. 78 (2013), 869-894.

[9] J. García-Luengo, P. Marín-Rubio and J. Real, Pullback attractors for three-dimensional non-autonomous Navier-Stokes-Voigt equations, Nonlinearity 25 (2012), 905930.

[10] M. Holst, E. Lunasin and G. Tsogtgerel, Analysis of a general family of regularized Navier-Stokes and MHD models, J. Nonlinear Sci. 20 (2010), 523-567.

[11] V. K. Kalantarov and E. S. Titi, Global attractors and determining modes for the 3D Navier-Stokes-Voight equations, Chin. Ann. Math. Ser. B 30 (2009), 697-714.

[12] C. J. Niche, Decay characterization of solutions to Navier-Stokes-Voigt equations in terms of the initial datum, J. Differential Equations 260 (2016), 4440-4453.

[13] A. P. Oskolkov, The uniqueness and solvability in the large of boundary value problems for the equations of motion of aqueous solutions of polymers, Zap. Nauchn. Sem. Leningrad. Otdel. Mat. Inst. Steklov. (LOMI) 38 (1973), 98-136 (in Russian).

[14] G. Yue and C. K. Zhong, Attractors for autonomous and nonautonomous 3D NavierStokes-Voight equations, Discrete Contin. Dynam. Systems Ser. B 16 (2011), 9851002.

[15] C. Zhao and H. Zhu, Upper bound of decay rate for solutions to the Navier-StokesVoigt equations in $\mathbb{R}^{3}$, Appl. Math. Comput. 256 (2015), 183-191.

Cung The Anh (corresponding author)

Department of Mathematics

Hanoi National University of Education

136 Xuan Thuy, Cau Giay

Hanoi, Vietnam

E-mail: anhctmath@hnue.edu.vn
$\mathrm{Vu}$ Manh Toi

Faculty of Computer Science and Engineering

Thuyloi University 175 Tay Son, Dong Da

Hanoi, Vietnam

E-mail: toivmmath@gmail.com 
